\title{
Christine Bergé, L'Odyssée de la mémoire
}

Paris, La Découverte, coll. « Les empêcheurs de tourner en rond », 2010, $271 \mathrm{p}$.

\section{Daniel Vidal}

\section{(2) OpenEdition} Journals

\section{Édition électronique}

URL : http://journals.openedition.org/assr/22873

DOI : $10.4000 /$ assr.22873

ISSN : $1777-5825$

Éditeur

Éditions de l'EHESS

\section{Édition imprimée}

Date de publication : 31 décembre 2011

Pagination : 115

ISBN : 9782713223273

ISSN : 0335-5985

\section{Référence électronique}

Daniel Vidal, «Christine Bergé, L'Odyssée de la mémoire », Archives de sciences sociales des religions [En ligne], 156 | octobre-décembre 2011, document 156-20, mis en ligne le 14 février 2012, consulté le 21 septembre 2020. URL : http://journals.openedition.org/assr/22873 ; DOI : https://doi.org/10.4000/ assr.22873

Ce document a été généré automatiquement le 21 septembre 2020.

(c) Archives de sciences sociales des religions 


\section{Christine Bergé, L'Odyssée de la mémoire}

Paris, La Découverte, coll. « Les empêcheurs de tourner en rond », 2010, $271 \mathrm{p}$.

\section{Daniel Vidal}

\section{RÉFÉRENCE}

Christine BERGÉ, L'Odyssée de la mémoire, Paris, La Découverte, coll. « Les empêcheurs de tourner en rond », 2010, 271 p.

1 Une anthropologie de la mémoire est-elle possible, qui suppose la mobilisation de concepts strictement contextualisés, une analyse affinée et ouverte des techniques et dispositifs de recherche qui ont, dès l'Antiquité, "modélisé » la question mémorielle, et une exploration rigoureuse des conditions et ratages de l'activité mnémonique ? D'une élaboration toujours vigilante, d'une écriture toujours passionnée et rebelle à toute clôture, l'ouvrage de Christine Bergé est à ce jour la meilleure réponse à cette interrogation. Il est le récit d'une conquête, peu à peu, du territoire de la mémoire comme activité permanente de survie de l'être humain en son identité, ses malfaçons, ses faillites, ses bonheurs-la-chance. Sa dramaturgie. Que la mémoire, comme toute autre instance de pensée, se définisse comme construit socio-technique, qu'elle témoigne donc, en chacune de ses formulations, de la singularité historique qui les autorise, cela ne la dispose pas pour autant comme signifiant assez flottant pour valoir joker sollicité pour combler quelque lacune dans l'arsenal métaphysique. Non plus que comme catégorie close en elle-même, que chaque nouvelle recherche déconstruirait pour en dessiner une nouvelle carte et une nouvelle fonction. D'une certaine façon, l'auteure, remontant aux sources impossiblement lisibles de la mémoire, au travers des techniques qui ont tenté d'en dire la raison et ses effondrements, fait à proprement parler, par là même œuvre de mémoire à son tour. C'est en ce repliement de l'acte de recherche sur l'objet de la quête que tel ouvrage, en tous ses moments, tient sa capacité 
de conviction. Remonter le cours de la mémoire jusqu'à ses ancrages organiques, collectifs, existentiels est se faire mémoire soi-même, comme si, remontant un fleuve, tel Alphée, l'on se faisait fleuve à son tour. Ainsi qu'après un long périple, Ulysse retrouve Ithaque et son lit nuptial... comme une mémoire reconquise.

D'emblée, l'essai pose la mémoire comme foyer de questions souveraines: est-il définition plus exacte de la pensée que la capacité, pour chacun d'entre nous, de se souvenir, d'oublier, et, par là même, d'être? La mémoire n'est-elle pas l'aptitude à cacher un secret au plus profond de soi, au risque d'être en souffrance sans cesse, mais condition aussi bien de survivre? Mémoire est "volonté de vivre", écrit l'auteur, « renouvelant à chaque instant l'affirmation d'être ». Mémoire chevillée au corps, mais comment saisir cet ancrage, comment penser cette aptitude singulière de l'être humain à recomposer en permanence, comme en sommeil paradoxal, sa " mémoire génétique ", qui, à vrai dire, définit très rigoureusement ce qui, en tel être, est l'humain en majesté ? L'auteure interpelle la mémoire et la poursuit en ses ultimes retranchements - là où, précisément, quelque chose d'elle est retranché du souvenir, quelque part en l'esprit retenu, en souffrance de n'être pas dicible, et cependant frayant désespérément sa voie pour parvenir aux frontières du monde et son écoute. Car la mémoire est bien ceci : un incessant travail de restauration identitaire en même temps qu'un repérage des failles et échecs d'une souvenance parfaite. Au demeurant, pourrait-on concevoir un esprit entièrement saturé de souvenirs, d'impossibles oublis, d'égale présence à tout événement de sa vie singulière? Oui, cela est concevable: tel esprit entre en cette étrange posture de se constituer malade afin de pouvoir revendiquer le droit irrévocable à l'oubli. La mémoire est ainsi cette extraordinaire machinerie qui, à raison même du "principe de déchiffrage, de conservation et de persévérance concernant l'être humain ", autorise une nouvelle conception de la "maladie mémorielle »: non plus, comme il est convenu de la qualifier, une désorganisation de l'esprit, mais un " travail de restauration, de compensation de l'individu affecté ». Si bien que, pour des auteurs comme Oliver Sachs ou Marvin Minsky, dont l'auteure rappelle les travaux pionniers, le champ de la mémoire est le champ même où se déploient, et se déplorent, les plus graves dommages - pertes, effondrements, secrets, enfin, qu'il convient de revivre afin que sur soi-même la " vérité » soit dite, et reconnue.

On voit combien toute recherche sur la mémoire est aussitôt mise au clair de sa complexité, et de la pluralité de ses significations. Et le «moi » n'est plus alors que le signifiant fallacieux d'une identité multipliée. Les technologies de la mémoire ont ainsi, au cours des siècles, exploré le labyrinthe de l'«esprit», cet autre nom de l'acte mémoriel. En l'explorant, elles ont pris le risque de rater l'« objet » de recherche au bénéfice d'un artefact. L'auteur est consciente de ce risque, qu'elle identifie sans en disqualifier les aboutissants : car ce furent paris à courir, chaque moment de la quête répondant à une conjoncture du savoir. Il fallait, pour cela, en finir avec la conception du sujet comme "machine sans devenir", dont le langage serait cette "structure profonde et universelle» que l'individu ne ferait qu'actualiser en fonction de son inscription dans un «fragment d'histoire et d'idéologie». Au revers de cette conception déterministe et totalisante, le travail de, et sur, la mémoire découvre un multivers sibyllin, infiniment plus proche d'Artaud et de Jankélévitch, que de Chomsky ou Althusser...

4 Car il en va de la mémoire comme d'une "partition ", propre à "harmoniser des systèmes hétérogènes dans l'espace et le temps ", et sachant prendre en compte ces 
lapsus de la temporalité, ces écroulements, ces césures, qui définissent la mémoire comme réseau de perte plus que raison de gain. En son commencement, la mémoire fait l'homme. Mais qu'entend-on par commencement? L'archéologie de la mémoire achoppe inévitablement à cette question sans réponse possible. C'est en quoi elle est la seule pertinente : l'origine est ce « point aveugle » à partir duquel il convient de partir, pour tenter d'y refluer ensuite. "Aveugle», en ce qu'il est le "point zéro » toujours précédé d'un négatif à l'infini : et c'est ce passage du " négatif » au "positif » qui est à proprement parler l'« origine ». Car c'est ici, en ce temps-rupture, en ce temps décisif, que mémoire est ce « cœur chimique » où $O$. Sachs demande que l'on plonge. Là, que tout, ou l'essentiel, se joue. Où, plus précisément, tout s'enchaîne. Ou se déchaîne. Temps immémorial parce que temps suspendu à quelque crochet, croche-pied, de l'histoire personnelle. Et c'est bien évidemment ce temps hors de toute loi qui va façonner à sa manière la mémoire que nous tentons à chaque instant de réparer par nous-mêmes. Ou par la médiation d'un tiers.

Car ce temps interrompu, loin d'être temps mort, est mis en veilleuse pour des catastrophes à venir, de corps et d'esprit, qui font retour - et dans une présence alors de grande violence -, comme l'empreinte d'un chaos d'histoire personnelle ou de lignée familiale devenant «tout à coup visible». Quel «sujet " retient cette mémoire en faillite, jusqu'à ce qu'elle vienne au cœur d'une identité de parade, et la bouleverse ? De quelle économie psychique/organique relève cette redoutable réserve de souvenirs abolis ? La mémoire est bien cela : souvenance et secret, souvenance parce que secret. Mais quand le secret, d'impossible symbolisation, ou sublimation, fait irruption "diabolique » dans la vie de l'être humain, il faut alors en connaitre la " raison ", pour en maîtriser le cours. Il faut, écrit l'auteure, " arrêter le temps et opérer un retour sur soi », car se souvenir, loin d'être un acte simple et paisible de re-connaissance, suppose un travail de " déconstruction » du soi, et de mise à nu de ce qui, en ce soi, est signature de sang. Si Mnémosyne est mère des arts et des sciences, mère des Muses ordonnant le monde et l'immonde, alors la mémoire est bien ce qui fait l'être humain véritablement homme, en sa singularité. Ainsi, Ch. Bergé rappelle que l'Antiquité définissait la mémoire comme capacité à «organiser les éléments en une dramaturgie personnalisée », et qu'Aristote liait toute mémoire à une éthique, engageant ainsi « la maitrise du destin moral ", cet autre nom de l'esprit tout entier lové autour de la révélation de son secret, qui peut être sa faute, ou quelque faillite collective venant s'y échouer.

En ce sens, le rêve vaut invention d'un nouveau monde, et mise au net de ce que secrète un esprit en son inquiétude. Descartes ne rêve pas impunément, si l'on peut dire, et l'auteure y lit une « technique naturelle de refondation et de métamorphose accomplie en l'absence du sujet conscient». Car le sommeil de la raison n'enfante pas que des monstres : il libère la voie à la démonstration. De là la méthode et son discours, de là la " méditation ", à partir d'un " oubli radical » qui permet à Descartes de reconstruire le monde et ses raisons. Il y a plus : les rêves du philosophe s'engrènent et font récit, cet "outil mnémotechnique » qui intègre les éléments symboliques en des "ensembles interprétatifs » de plus vaste ampleur. La mémoire serait-elle alors une mise en scène des événements survenus - à douleur à plaisir - aussi loin que l'on s'en souvienne, qui seule puisse permettre d'oublier ceux que l'on veut laisser pour compte ? Jusqu'à ce qu'il faille un jour, qui peut être une vie entière, régler précisément ce compte? Aussi bien la mémoire est-elle à double sens : instance de créativité sous condition d'abolition de soi (dans le rêve), et creusement d'un abîme au centre de l'existence. Cet abîme n'est 
pas perdition. Analysant l'économie du somnambulisme, et les pratiques du spiritisme, l'auteure nomme ce qui, en soi, est l'autre: il est «l'oubli d'un moi remisé dans les arrière-fonds et pourtant détenteur des puissances de l'individu ». À mémoire duelle, individu capable "de vivre sur deux scènes en même temps». Que telle vie soit pensable, chamanes, extatiques, spirites en témoignent. Et ouvrent ainsi, au centre de la mémoire, un champ vertigineux d'interrogations, que ne cesseront d'explorer les différentes écoles de psychologie, et ce qui apparaît aujourd'hui comme leur couronnement et leur implosion, les psychanalyses - Freud, Jung, Férenczi.

Il faut, pour que mémoire soit, traverser en effet la frontière entre le dicible et ce que le trauma a figé dans une zone de futures tempêtes. C'est ce « rapport de soi à soi » que les technologies de la mémoire ont pour tâche de restaurer. Ainsi opèrent ces passeurs, virtuoses des désarrois de l'esprit, "médiateurs d'un théâtre de l'âme ", faisant venir au jour ces souffrances et leur cortège de visions, de paroles défuntes, de transes, de possessions, de convulsions. Comme il en va des corps, les "âmes" sont "tatouées", saturées de signes à déchiffrer, témoins d'une «vie intérieure (...) indomptée et immuable »- foyer, selon la formule heureuse de l'auteure, de « mémoires occultes ». Il s'agit alors, spirites ou analystes, et chacun selon des procédures distinctes, de « jouer sur les franges du soi », pour tenter d'accéder, «patient » et « analysant » emportés par une même obligation de quête, au noyau de cette amnésie qui interdit le souvenir, et la survie qui va avec. Sommeil somnambulique, analyse des "profondeurs", technique spirite, hypnose, libres associations sur le mode du "cadavre exquis»: chaque expérience d'écoute et de travail au plus près du «deuil» vaut engagement en un "voyage dans l'altérité", où l'autre est soi-même redoublé. Faut-il remonter à la « scénique des origines »? Oui, rappelle l'auteure, car là, en cette origine aveugle/ aveuglante, est le « site des réminiscences ». Et là se dédouble la mémoire et ce qui fait l'humain dans l'homme désormais séparé.

Il est à profusion des signes de cette altérité/altération. Stigmates, « métamorphose des douleurs en une surface de mémoire ", lorsque la peau est de chagrin disant la douleur christique, ou la grande colère de Dieu. Sommeils à l'emporte-corps, qui déroulent une mémoire comme "palimpseste d'histoire présente et passée ", et qui vivent et dénouent leur drame comme trame tissée qui libère et renoue du sens au lieu même qui n'était que perte et oubli. "Écritures carnées ", que tentent de saisir les arts nouveaux de la photo et de capter les myographes, ces "sismographes de l'âme »- et ce sont écritures de sang, écritures de « foudre ». Ou de « lumière », en la mystique, cet " art de la mémoire » de la Passion, ré-engendrant continûment la formule du sacré. En une dévoration du temps, qui institue la mystique comme extrême compression des durées : passé et présent ne sont plus qu'un - il n'est, à tout prendre, rien qui ne soit de pur présent. Pour rendre compte de cette véritable collision des temporalités, sans doute convient-il de "créer une simultanéité artificielle", pour "pénétrer au cœur d'un temps qui est bloqué ». Mais ce que propose ici l'hypnose vaut, par delà la méthode, pour toute autre technique de réfection mémorielle. Les " écritures moléculaires », qui codent, dans la double hélice de l'ADN, «l'échelle immémoriale des acquisitions vivantes " nous parlent à coup sûr de ce qui est inné en l'homme, mais où l'inné peut apparaître comme l'agencement ultime de l'acquis... Ainsi comprend-on mieux le paradoxe de toute quête de l'origine, à la fois plongée dans le tréfonds du biologique, et déjà codée par la traversée des expériences de toute vie. 
9 Alors, la mémoire? Traces indélébiles, héritages de fantômes habitant nos nuits et nos jours, douleurs du passé ? L'essai rassemble le faisceau de ces écritures revendiquées et de ces blessures déniées, de ces brusques remontées de temps que l'on croyait à jamais révolus, de ces interdits que l'on pensait intangibles, mais qu'en sourdine l'on savait transgressés, et en dit la mémoire, ce carrefour des temporalités. La mémoire, ou le double fléchage du temps, qui est en vérité sa grammaire: "de l'imparfait», écrit l'auteure, et "d'un futur antérieur ». Passé - tissu de traces « inaccessibles »; avenir déjà destiné - où ces mêmes traces s'inscrivent en « toile mouvante » que l'on compose et recompose sans cesse. La mémoire est bien dès lors ce « récit » traversé de toutes les failles et les fautes par quoi l'homme tente de régler quelque « dette » envers un passé inavoué, "à la recherche du trauma perdu», qui fut sa perte essentielle. Mais un récit écrit, si l'on peut dire, à deux mains, ou, si l'on veut, à deux sujets : l'analyste, note S. Ferenczi, doit " être transporté avec le patient dans cette partie de son passé », afin, explicite l'auteure, que le patient " puisse croire à la réalité de l'événement ». Pas plus qu'il n'est de mémoire hors de l'histoire existentielle de chacun, il n'est de mémoire solitaire.

10 Tel est sans doute le secret de toute " guérison » des maladies ou intermittences de la mémoire, au rebours de toute apparition spectrale ou de tout dédoublement de l'esprit: la "survie psychique » est au prix d'une parole enfin parvenue à se faire entendre, et disposée alors dans un réseau social, fût-il minimal, qui accepte d'en partager l'offrande. L'Odyssée de la mémoire nous dit bien cela : la guerre de Troie a bien eu lieu, trauma/ mythe décisif, et long fut le voyage du retour, comme une mémoire sans fin, passant par le royaume des morts, jusqu'à ce que le sujet/héros soit enfin reconnu, avec ses cicatrices et sa puissance exacte. Il fallait cette aventure de l'esprit pour que revienne la mémoire, et qu'une écriture en porte témoignage. De toute première qualité est celui que nous propose Christine Bergé en son ouvrage sur la mémoire, sombre océan pour sombre oubli. 